\title{
Coût d'opportunité versus coût du maintien des potentialités écologiques : deux indicateurs économiques pour mesurer les coûts de l'érosion de la biodiversité
}

\author{
Harold Levrel ${ }^{1}$, Julien Hay ${ }^{2}$, Adeline Bas ${ }^{3}$, Pascal Gastineau ${ }^{4}$, Sylvain Pioch ${ }^{5}$ \\ ${ }^{1}$ Économiste, IFREMER, UMR AMURE, 29280 Plouzané, France \\ 2 Économiste, Université de Brest, UMR AMURE, 29334 Quimper cedex, France \\ ${ }^{3}$ Économiste, Université de Brest, UMR AMURE, 29280 Plouzané, France \\ 4 Économiste, IFSTTAR, Laboratoire Transport et Environnement, 69675 Bron cedex, France \\ ${ }^{5}$ Biogéographe, Université Paul Valéry, Montpellier 3, UMR 5175 CEFE, 34199 Montpellier Cedex 5, France
}

Il existe deux méthodes pour évaluer le coût del'érosion de la biodiversité : calculer la valeur monétaire de cetteérosion ou calculer le coût du maintien des potentialités écologiques pour compenser cette érosion. Les auteurs proposent dans cet article d'évaluer les forces et les faiblesses de ces deux approches, en soulignant comment et pourquoi la seconde semble bénéficier aujourd'hui d'un intérêt grandissant dans la réglementation. L'analyse qu'ils proposent permet aussi d'insister sur la nécessité de prendre du recul par rapport à la tendance croissante à assimiler les processus biologiques aux services écologiques, puis ces derniers aux services économiques, finalement objets de politiques publiques.

Cet article fait suite aux questions posées par Jean-Michel Salles (NSS, 18, 4 [2010]) sur la signification de l'évaluation économique de la biodiversité et des services écosystémiques. Il est à rapprocher de celui de Philippe Méral publié dans ce même numéro.

La Rédaction

\section{Mots-clés :}

services écologiques ; biodiversité ;

valeur ;

coûts ;

méthodes d'estimation
Résumé - La question des coûts de l'érosion des services écologiques est un sujet très important, comme l'a souligné le programme TEEB (The Economics of Ecosystems and Biodiversity), qui a proposé une évaluation des coûts de l'inaction politique dans le domaine de la conservation de la biodiversité. Dans la même veine, le rapport du Centre d'analyse stratégique, intitulé Approche économique de la biodiversité et des services liés aux écosystèmes, s'est intéressé à la question de la valeur des services écosystémiques et a proposé quelques éléments qui permettraient d'évaluer le coût de leur érosion. Il existe cependant une certaine confusion autour de cette notion de coût. Ainsi, dans le cas du TEEB, l'évaluation proposée des coûts traduit en termes monétaires la perte de bénéfices potentiels que les services écologiques perdus auraient pu délivrer à l'horizon 2050. Mais une autre manière d'évaluer ces coûts aurait pu être d'estimer les investissements à réaliser pour maintenir les capacités écologiques des écosystèmes à délivrer ces services. En approfondissant ce point, le présent article permet de souligner les différences entre les implications opérationnelles de ces deux approches, mais aussi leurs connexions avec des processus décisionnels précis. Une de nos conclusions est que l'approche économique de la biodiversité a tendance à se focaliser systématiquement sur la question de la valeur monétaire des services écologiques, alors que l'évaluation des coûts de maintien des potentialités écologiques dont dispose la biodiversité pour délivrer des services écosystémiques semble être aujourd'hui privilégiée dans les textes réglementaires.

\section{Keywords: \\ ecosystem services; biodiversity; value; \\ costs; assessment methodology}

\begin{abstract}
Opportunity cost vs maintenance cost of ecological potential: two economic indicators for measuring the cost of biodiversity decrease. The cost of ecosystem services degradation is a major topic as underlined by the TEEB program (The Economics of Ecosystems and Biodiversity), which proposed an assessment of the costs of policy inaction in the field of biodiversity conservation. In the same vein, the report of the French Centre for Strategic Analysis on the Economic Approach to Biodiversity and Ecosystem Services turns its attention to the value of ecosystem services, suggesting some elements that could help measure the cost of biodiversity degradation. There is however some
\end{abstract}

Auteur correspondant : Harold.Levrel@ifremer.fr 
confusion regarding the notion of cost. Thus, in the case of TEEB the proposed cost assessment translates into monetary terms the loss of potential profits from ecological services. Yet, another way of assessing these costs could be to estimate the investment required to maintain the ecological potential of ecosystems to deliver these services. This latter approach may be considered better suited to the concept of "inaction" proposed by the TEEB report. In addressing this point, the paper attempts to identify the operational implications of these two assessment methods and their links with decision-making. One of our findings is that the economic approach to biodiversity tends to focus systematically on the question of the monetary value of ecosystem services while regulatory texts increasingly consider the cost assessment of maintaining ecosystem services.

\section{Les coûts associés à l'érosion de la biodiversité : deux représentations possibles}

On sait depuis la dernière Conférence des Parties, qui a eu lieu à Nagoya en octobre 2010, que l’objectif fixé par la Convention sur la diversité biologique - ralentir le rythme de l'érosion de la diversité du vivant sur notre planète à $\mathrm{l}^{\prime}$ horizon 2010 -n'a pas été atteint. On sait aussi que cette érosion est assimilée à un coût pour nos sociétés, qui voient, à travers elle, un grand nombre de potentialités humaines disparaître (Millennium Ecosystem Assessment, 2005).

C'est tout à la fois pour prolonger le travail du Millennium Ecosystem Assesment (MA), pour rendre plus concrète cette perte sociale et économique, mais aussi pour porter la question de l'érosion de la biodiversité au même niveau que celle du réchauffement climatique dans les débats publics que le TEEB (The Economics of Ecosystems and Biodiversity) a été lancé en 2007 - et conclu en 2010, avec la remise de son dernier rapport lors de la conférence de Nagoya. Une tâche importante de ce programme a été d'évaluer les coûts de l'inaction politique à l'échelle mondiale, dans l'esprit du rapport Stern sur le réchauffement climatique (Braat et ten Brink, 2008).

On peut considérer schématiquement qu'il existe deux approches principales pour évaluer ces coûts. La première consiste à exprimer en termes monétaires la valeur des bénéfices fournis par la biodiversité à l'homme, bénéfices non marchands pour une grande part et qui sont perdus du fait de l'érosion de cette dernière (Chevassusau-Louis et al., 2009 ; Barbier et al., 2009). La seconde est d'évaluer le coût de mise en œuvre des investissements nécessaires au maintien du flux de services écosystémiques fournis par la biodiversité (Bartelmus, 2009; Weber, 2007). Ainsi, dans le cas d'une pollution, il est possible d'inférer deux valeurs monétaires d'un dommage environnemental: d'une part, la perte de bénéfices engendrée par le dommage ; $d$ 'autre part, les coûts de restauration à engager pour recouvrer les services perdus à la suite de ce dommage. On peut visualiser les différences qui existent entre ces deux valeurs monétaires à partir du cadre logique du Millennium Ecosystem Assessment (Fig. 1).

La flèche qui va de la boîte « biodiversité » à la boîte « bien-être " est aujourd'hui principalement envisagée à partir d'une évaluation monétaire. En effet, même si les travaux récents s'intéressant à l'approche économique de la biodiversité et des services écologiques soulignent systématiquement l'existence d'indicateurs alternatifs pour évaluer la contribution des services écologiques au bienêtre humain, ces travaux privilégient souvent, in fine, l'évaluation monétaire en tentant d'affecter un prix aux différentes catégories de services écologiques offerts par la biodiversité (Chevassus-au-Louis et al., 2009 ; Environmental Protection Agency, 2009 ; Braat et ten Brink, 2008). La flèche allant de la boîte « facteurs directs de changement » vers la boîte « services écosystémiques » nous dit tout autre chose. Elle cherche à souligner que les usages de la biodiversité par la société ne sont pas seulement une source d'érosion pour cette dernière, mais aussi une source de potentialités. C'est le cas lorsque les sociétés humaines entreprennent des actions positives pour l'environnement naturel: restauration d'écosystèmes, contrôle de certaines espèces invasives, suivi de l'état de l'environnement pour en appréhender les changements, etc. (Jones et Pease, 1997 ; Dunford et al., 2004 ; Roach et Wade, 2006 ; Zafonte et Hampton, 2007 ; Levrel et al., 2010). À cette flèche peut aussi correspondre une évaluation monétaire, mais elle a pour objectif de mesurer le coût associé à la mobilisation de capital humain, naturel et physique pour le maintien ou l'accroissement des flux de services écologiques délivrés par la biodiversité. Ces investissements sont le plus souvent associés à des réglementations qui nécessitent de mettre en œuvre des actions positives en faveur de la biodiversité (loi sur la responsabilité environnementale, directive-cadre sur l'eau, Natura 2000, etc.). Mais ils peuvent aussi avoir une origine volontaire du fait d'incitations financières ou simplement morales.

La première approche s'intéresse ainsi aux équivalences monétaires et considère les services écologiques et la biodiversité comme des éléments constitutifs du bienêtre des individus. La seconde approche privilégie une 


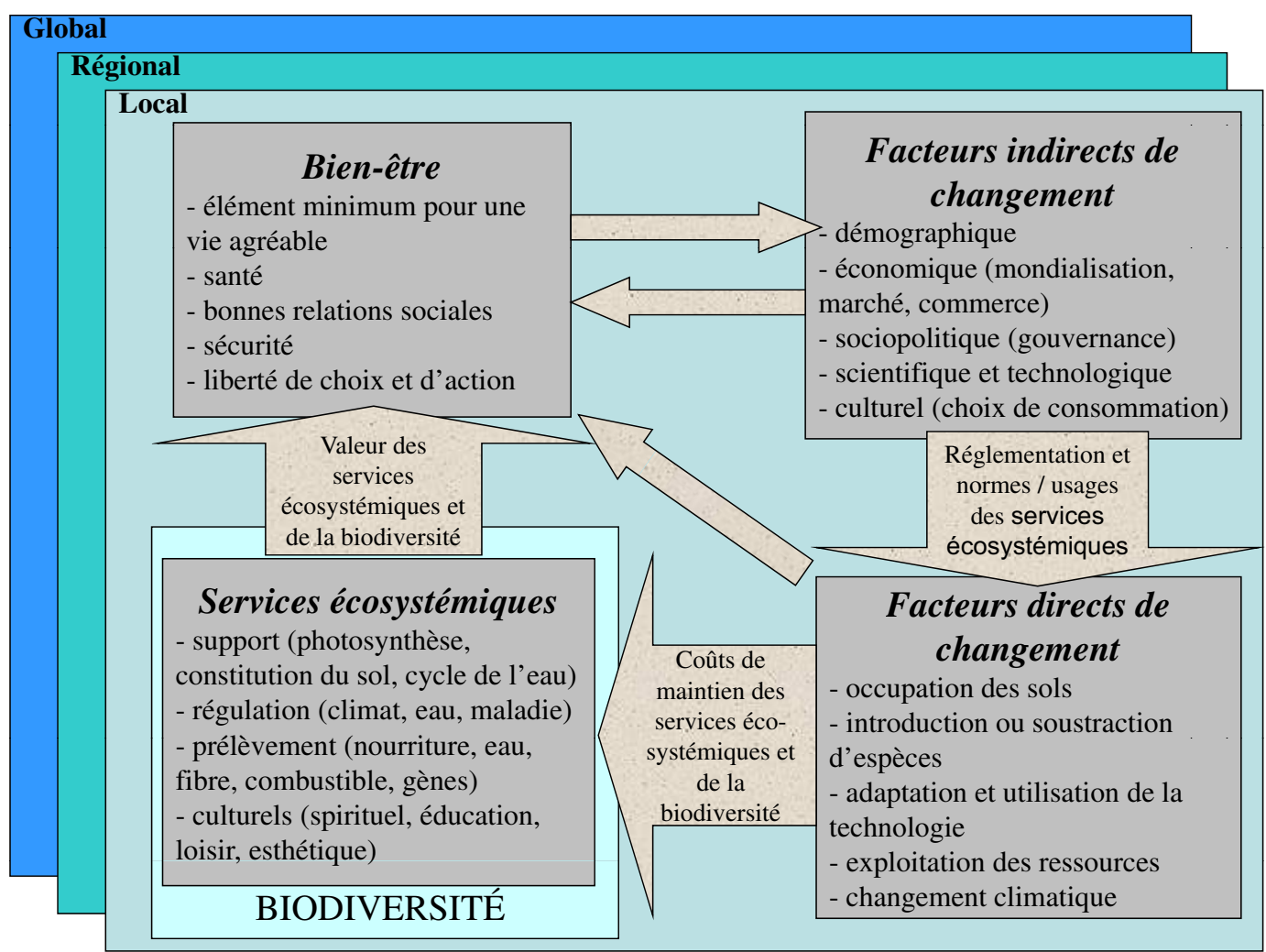

Fig. 1. Situation des deux modes d'évaluation dans le cadre logique du Millennium Ecosystem Assessment (schéma adapté à partir du Millennium Ecosystem Assessment, 2005).

démarche en termes d'équivalences physiques (services écologiques et biodiversité) et s'intéresse aux potentialités écologiques sans présumer, autant que la première, des liens plus ou moins explicites qui existeraient avec le bien-être humain. La première approche est donc plus anthropocentrée que la seconde.

Ajoutons que l'objectif n'est pas le même et que les démarches peuvent apparaître comme complémentaires. Dans le premier cas, on cherche à évaluer la perte de bénéfices associée à un constat d'érosion de la biodiversité ; dans le second cas, on propose une analyse des coûts relativement à un critère d'efficacité - le maintien de la biodiversité - qui prend davantage en compte les enjeux écologiques en relation directe avec des objectifs politiques et des normes réglementaires.

Dans cet article ${ }^{1}$, nous souhaitons analyser les implications opérationnelles de ces deux approches ainsi que leurs forces et leurs faiblesses.

\footnotetext{
1 L'idée de cet article est née d'un besoin de clarification de la manière dont les coûts de l'érosion de la biodiversité peuvent être calculés, dans un contexte où l'on observe une multiplication des programmes d'évaluation monétaire de la biodiversité.
}

\section{Les coûts de l'érosion de la biodiversité et des services écologiques sous l'angle d'une perte de valeur économique}

\section{Le principe de l'évaluation}

Les économistes aiment souvent rappeler la maxime " There is no free lunch », laquelle met en avant le fait que chaque décision contient invariablement un aspect négatif, considéré comme un coût (monétaire ou non, objectif ou subjectif). La décision d'inaction n'échappe pas à la règle. En effet, même si elle est intuitivement associée à la non-mise en œuvre de moyens nouveaux et donc à un coût additionnel nul, la décision d'inaction implique nécessairement un coût sous forme de pertes d'opportunités associées à des décisions alternatives.

Le TEEB s'est concentré sur cette évaluation des coûts d'opportunités associés à l'inaction politique dans le domaine de la conservation de la biodiversité, en estimant les bénéfices rendus par les écosystèmes auxquels la collectivité renonce en affectant ses ressources disponibles à un usage alternatif. C'est l'approche qui avait été adoptée précédemment par le rapportStern, faisantétat du coût de 


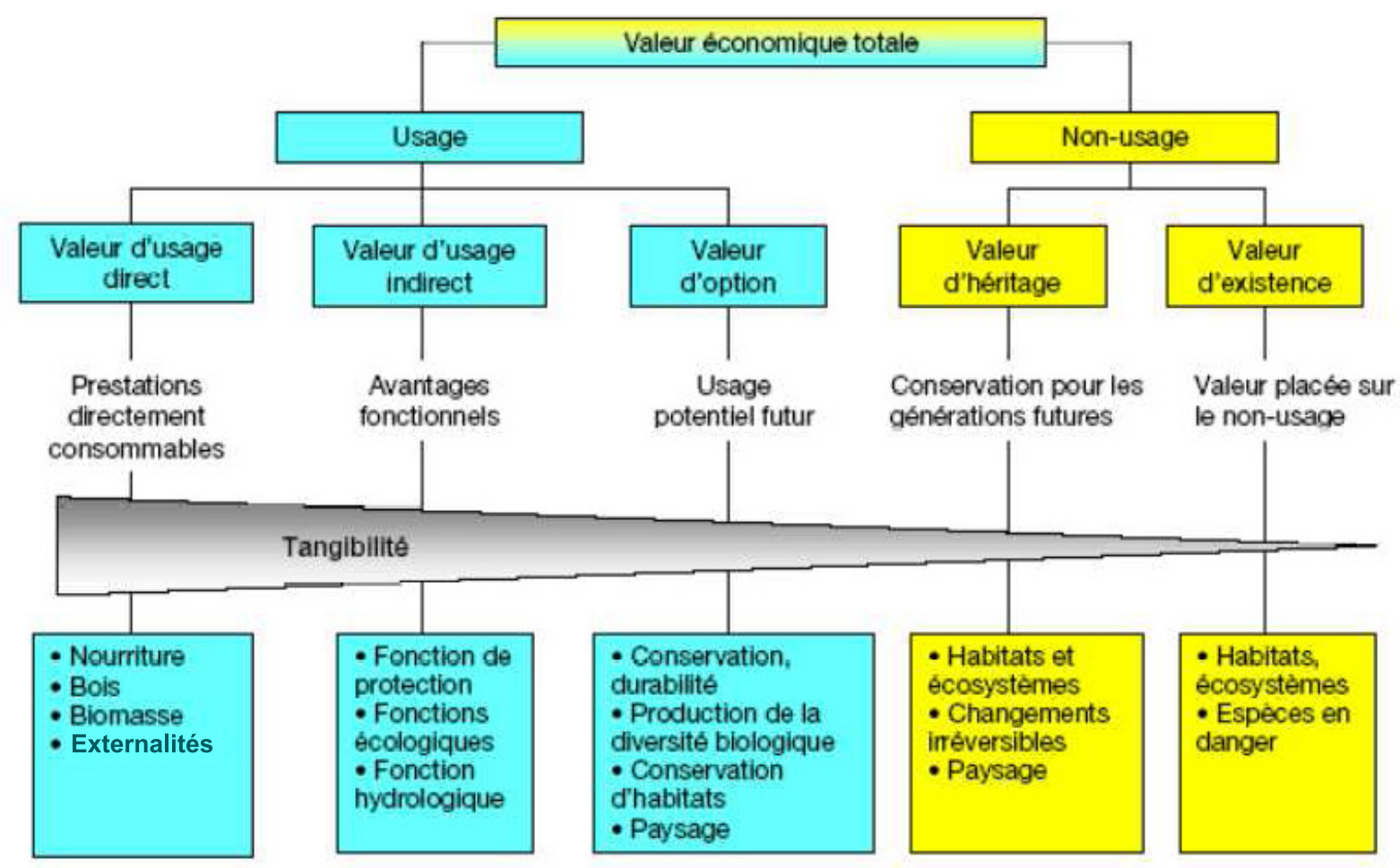

Fig. 2. Description des différents éléments de la valeur économique totale de l'environnement (source : Chevassus-au-Louis et al., 2009, p. 174).

l'inaction dans le domaine du réchauffement climatique. Les indicateurs de coûts évalués dans le cadre du TEEB représentent ainsi le manque à gagner, en termes de bienêtre social, correspondant à un scénario d'inaction en faveur de la biodiversité (Braat et ten Brink, 2008). Le coût de cette inaction est évalué à partir d'un scénario selon lequel le rythme de l'érosion de la biodiversité observé aujourd'hui se poursuit au cours des quarante prochaines années. Il s'agit donc d'un scénario de statu quo, nommé «business as usual », qui permet de souligner quelle va être la perte de bénéfices associée à l'érosion des services écologiques si on ne fait rien au cours des prochaines années. L'estimation des coûts de l'inaction politique proposée par le TEEB s'élève à 13938 milliards d'euros pour la période 2000-2050 (ibid.).

Le TEEB n'est pas le premier travail à proposer une évaluation monétaire de la biodiversité à une échelle spatiale large, sinon mondiale. Ainsi, dix ans plus tôt, Costanza et al. (1997) proposaient-ils déjà une estimation de la valeur des flux annuels des services écologiques offerts par la biosphère à l'homme. Ces flux étaient estimés à 33000 milliards de dollars. L'objectif n'était pas alors d'évaluer les coûts de l'inaction à proprement parler, mais de souligner l'importance de la valeur économique totale ${ }^{2}$ (Fig. 2) des services écologiques fournis à l'homme, en proposant un ordre de grandeur pour cette dernière.
Ce type d'évaluation a ensuite donné lieu à des déclinaisons par écosystème. À titre d'exemple, on rappellera qu'en France le Centre d'analyse stratégique (CAS) a proposé une évaluation des services écologiques délivrés par les forêts françaises, qui serait de 970 euros par hectare et par an pour une valeur actualisée totale équivalente à 35000 euros (Chevassus-au-Louis et al., 2009).

Un autre exemple est le travail réalisé par Martinez et al. (2007) - à partir de la même méthode que celle de Costanza et al. (1997) -, qui estime la valeur annuelle des services écologiques fournis par les écosystèmes côtiers à 2102 milliards de dollars. Cette étude, qui est déclinée par pays, donne une estimation de 28 milliards pour la France, dont près de 13 milliards pour la NouvelleCalédonie et 10 milliards pour la Métropole.

\footnotetext{
2 De manière schématique, les valeurs qui composent la valeur économique totale peuvent être séparées en deux grandes catégories : celles relatives à des usages et celles relatives aux non-usages. Les valeurs d'usage renvoient à une utilisation directe ou indirecte, marchande ou non marchande, voire à une valeur d'option reposant sur des usages potentiels futurs (Heal, 2000). Les valeurs de non-usage reposent essentiellement sur le principe que la biodiversité a une valeur pour elle-même et/ou représente un patrimoine pour les générations futures, même si elle est « ordinaire » (Krutilla, 1967).
} 
Tous ces chiffres sont le plus souvent comparés avec les PIB des pays ou du monde, de manière à souligner l'importance de ces services pour l'économie mondiale et, par conséquent, l'intérêt qui existe à investir dans la biodiversité. Ainsi, on peut observer que la valeur des services écologiques côtiers français, obtenue à partir des travaux de Martinez et al. (2007), est à peu près équivalente au PIB associé à l'économie maritime française, qui était pour la même année 2007 de 21,5 milliards d'euros (Kalaydjian, 2007).

Le problème, pour les tenants de ces évaluations, est qu'il n'existe pas, pour l'instant, de mécanismes financiers permettant d'intégrer ces valeurs dans les échanges marchands, ce qui conduit à la non-prise en compte de la plupart des services écosystémiques dans les stratégies d'investissement et les projets de développement adoptés par des opérateurs privés ou publics (Daily et al., 2009). $\mathrm{C}^{\prime}$ est ce déficit institutionnel qui justifierait le manque d'actions actuel et l'érosion de la biodiversité à laquelle nous assistons.

Cependant, au-delà de cette difficulté institutionnelle, on peut noter que l'approche par les coûts d'opportunités nécessite en premier lieu de pouvoir proposer une évaluation monétaire des services délivrés par la biodiversité, si l'on veut pouvoir estimer la perte cumulée des bénéfices qu' elle fournit à l'homme à l'horizon 2050. Il est donc important de revenir sur les méthodes qui permettent de proposer une telle évaluation monétaire.

\section{Les méthodes d'évaluation}

La plupart des méthodes d'évaluation économique sont fondées sur le cadre conceptuel utilitariste, dominant dans l'analyse économique. Cette approche repose sur la notion de substituabilité, qui permet d'inférer une valeur économique reflétant l'attachement des individus aux différents biens et services auxquels ils ont accès. John Hicks, un des pères de l'économie néoclassique moderne, évoque ainsi les mesures de surplus compensés associées à la substitution de différents biens entre eux et qui permettent, in fine, de conserver le même niveau d'utilité pour le consommateur ${ }^{3}$.

Nombre de services rendus par les écosystèmes ne font l'objet d'aucune transaction marchande et ne peuvent, pour cette raison, être mesurés en termes monétaires à partir de prix du marché. Aussi différentes méthodes ont-elles été développées par certains économistes pour tenter, malgré tout, d'attribuer de manière plus ou moins directe une valeur monétaire à ces services écosystémiques : évaluation contingente, analyse conjointe, coûts de déplacement, prix hédoniques, pour ne citer que les

3 Il s'agit de l'origine du principe de consentement à payer pour bénéficier de l'accès à certains biens et services substituables entre eux. plus connues (Environmental Protection Agency, 2009; Barbier et al., 2009). Ces méthodes sont aujourd'hui largement pratiquées et stabilisées dans le domaine de l'environnement. Pour autant, leur utilisation dans le domaine de la biodiversité nécessite de prendre quelques précautions. En premier lieu, s'il est souvent considéré par la littérature que les services écosystémiques sont bien monétairement évaluables, cette approche ne semble pas appropriée à certaines composantes de la biodiversité au motif qu'elles seraient non substituables (Heal, 2000 ; Barbier et al., 2009 ; Norgaard et Bode, 1998). Le deuxième niveau de précaution concerne les capacités à calculer une valeur économique totale (Fig. 2) des composantes retenues, sans laquelle l'évaluation ne fera que sous-estimer la valeur réelle des services écosystémiques correspondants. En effet, s'il est aujourd'hui admis que l'on puisse approcher de manière fiable les valeurs d'usages direct et indirect de nombreux services écosystémiques délivrés par la biodiversité - lorsque les données existent -, les valeurs de non-usage ou celles associées aux processus élémentaires de reproduction du vivant sont en revanche plus délicates à estimer pour des raisons techniques - valeur moins tangible et peu familière aux yeux des individus - et éthiques - peut-on donner une valeur aux éléments constitutifs de la vie sur Terre (Norgaard et Bode, 1998) ?

Selon certains économistes, une manière de contourner cette limite est de ne pas retenir les valeurs de nonusage dans les évaluations et de simplement chercher à renseigner la valeur de services écosystémiques spécifiques, bénéficiant à des acteurs économiques précis, à une échelle spatiale et temporelle circonscrite (Heal, 2000 ; Barbier et al., 2009). Le gain ainsi obtenu du point de vue de la fiabilité des évaluations conduit cependant à abandonner l'objectif d'agrégation des valeurs et d'obtention d'une valeur économique totale.

\section{Implication opérationnelle de l'usage des coûts d'opportunités pour l'évaluation des coûts de l'érosion de la biodiversité}

Les points indiqués ci-dessus ne sont pas sans implication sur le plan opérationnel, et plus particulièrement sur la capacité à attribuer des valeurs monétaires robustes à la biodiversité et à son érosion. Comme nous venons de le mentionner, il est possible de proposer des évaluations monétaires des services écosystémiques à partir du moment où un certain nombre de précautions méthodologiques sont prises et lorsque les conditions dans lesquelles ces évaluations vont être véritablement opérationnelles sont bien circonscrites. Ce type d'évaluation serait donc envisageable lorsque : les services évalués sont clairement discriminés les uns vis-à-vis des autres ; ces derniers sont associés à des usages directs ou indirects ; le contexte socioéconomique et décisionnel dans lequel s'insère cette évaluation est précisé ; les processus 
écologiques sous-jacents à la production des services en question sont connus ; les techniques d'évaluation mises en œuvre sont validées par des expérimentations (Heal, 2000 ; Committee on Assessing and Valuing the Services of Aquatic and Related Terrestrial Ecosystems, 2004 ; Barbier et al., 2009).

Ces éléments contraignants permettent de comprendre pourquoi on obtient de très bonnes évaluations pour les services de prélèvement et, à l'opposé, des évaluations très critiquées pour les services de support (Heal, 2000). Entre les deux, les services culturels relatifs aux activités récréatives ainsi que les services de régulation nécessitent la mise en œuvre d'évaluations indirectes parfois délicates à réaliser. Enfin, les valeurs spirituelles associées aux services culturels, liés à la fois à la notion de biodiversité remarquable et à celle de valeur d'existence, $s$ 'avèrent très difficiles à estimer monétairement et finalement peu pertinentes, tant du point de vue technique que du point de vue éthique.

Par ailleurs, on peut s'interroger sur la légitimité, dans le cadre de choix collectifs, du " processus de commensuration " que représente l'évaluation de la valeur économique totale de la biodiversité, c'est-à-dire la réduction de la diversité des valeurs de la biodiversité à une unité de mesure unique (Espeland et Stevens, 1998 ; Desrosières, 2003). En effet, le débat public étant fondé sur la mise en interaction de différents systèmes de valeurs (Boltanski et Thévenot, 1991), on peut craindre que le processus d'évaluation monétaire n'implique une uniformisation qui puisse être dommageable aux négociations autour des enjeux de conservation de la biodiversité. Pourtant, malgré ces problèmes, la question de l'évaluation de la valeur économique totale de la biodiversité reste fortement d'actualité. Cela s'explique par le fait qu'il est nécessaire de calculer cette valeur économique totale pour pouvoir proposer des analyses coût-bénéfice pertinentes ${ }^{4}$.

Il existe cependant des pistes prometteuses qui offrent des alternatives à la valeur économique totale et à la prise en compte des valeurs de non-usage qu'elle nécessite. On peut mentionner le recours à des modèles permettant d'intégrer le rôle de la biodiversité dans les fonctions de production économique (Barbier, 1994 ; Costanza et al., 2007), voire de mettre en interaction différents types d'indicateurs de valeurs des services écosystémiques - écologiques, économiques et sociaux - et d'échapper ainsi aux deux problèmes-clés du calcul de la valeur économique totale que sont la monétarisation des valeurs de non-usage et l'agrégation de ces valeurs (Daily et al., 2009 ; Nelson et al., 2009).

\footnotetext{
4 En effet, si des arbitrages sont opérés en défaveur de la biodiversité sur la base d'une estimation partielle de sa valeur, les choix réalisés pourront être considérés comme discutables du point de vue économique.
}

\section{Les coûts de l'érosion de la biodiversité et des services écologiques à partir du coût de maintien des potentialités écologiques}

\section{Le principe d'évaluation}

En parallèle de l'évaluation des coûts d'opportunités associés à l'inaction politique, une seconde approche est possible, fondée sur les coûts du maintien de la biodiversité et des services écologiques qu'elle délivre (Dunford et al., 2004 ; Zafonte et Hampton, 2007). Il s'agit finalement, ici, d'évaluer les coûts de l'action dans un scénario de maintien des potentialités écologiques, qui bénéficieront à la fois à la biodiversité pour elle-même et aux usagers directs et indirects des services écologiques que celle-ci produit.

Cette méthode repose sur deux particularités qui la distinguent de l'approche précédente. La première, d'ordre technique, est de ne pas avoir à prendre en compte les incertitudes qui existent autour des valeurs réelles de la biodiversité et des services écosystémiques, en considérant que la restauration des écosystèmes permettra in fine de compenser toutes les pertes de bien-être subies à la suite d'une dégradation de l'environnement - à la condition que les populations qui bénéficieront de ces mesures de restauration soient les mêmes que celles qui ont subi le préjudice (Roach et Wade, 2006). La seconde, d'ordre éthique, est de respecter un critère de durabilité forte, qui repose sur l'hypothèse qu'une partie du capital naturel ne peut être substitué par du capital physique ou humain sans faire courir un risque d'effondrement au système écologique qui en est le support ${ }^{5}$ (Ekins, 2003). Dès lors, il s'agit d'évaluer les coûts du maintien de ces potentialités.

Il existe un certain nombre de travaux portant sur le coût de maintien de la biodiversité et des services écologiques, qui sont relatifs, pour la plupart, à des programmes de restauration ou de compensation (Borde et al., 2004 ; Fonseca et al., 2002 ; King et Bohlen, 1995 ; Lewis, 2001 ; Moberg et Rönnbäck, 2003 ; OCDE, 2004). Ces travaux peuvent être séparés en deux groupes : les programmes de restauration à large échelle et ceux focalisés sur des écosystèmes types. Pour les programmes à large échelle, on mentionnera les Everglades (7,8 milliards de dollars) [Borde et al., 2004], l'écosystème des

\footnotetext{
5 Cette approche s'oppose au principe de durabilité faible, qui postule une substituabilité parfaite entre les différentes formes de capital (Atkinson et Pearce, 1993 ; Banque mondiale, 1997). Du point de vue de la durabilité faible, le principe de «no net loss » écologiquen'a pas de sens. En effet, le seul « no net loss » qui compte est celui de la valeur économique fournie par les différentes formes de capitaux, quelle que soit l'importance relative de ces derniers.
} 
Catskills à proximité de New York (1,5 milliards de dollars) [National Research Council, 2000] ou la mer d'Aral (entre 30 et 50 milliards de dollars) [Micklin, 2007]. Pour les programmes focalisés sur des habitats types, les coûts de restauration varient grandement selon le niveau de dégradation qu'ils ont subi, le niveau de restauration recherché, les moyens mis en œuvre pour atteindre cet objectif et le contexte biophysique et réglementaire. Ainsi, du côté américain, le coût de l'hectare restauré à travers les procédures "Natural Resource Damage Assessment 》 varie de 18000 à 247000 dollars selon le type d'écosystème (OCDE, 2004).

En France, la Commission des comptes et de l'économie de l'environnement produit tous les ans un rapport sur les dépenses pour la protection de l'environnement sur le territoire national. En 2007, ces dépenses s'élevaient à 41,2 milliards d'euros, dont 1,54 milliards étaient dédiés à la biodiversité et aux paysages (Commission des comptes et de l'économie de l'environnement, 2009). Le CASévoque aussi dans son rapport (Chevassus-au-Louis et al., 2009) le coût de restauration par hectare d'une forêt de feuillus diversifiée dans une zone tempérée : il est évalué, en 2007, entre 5000 et 6000 euros. Cependant, il ne s'agit là que d'un plan de restauration fondé sur un programme de reboisement qui ne tient pas compte de l'ensemble des fonctionnalités écologiques liées à ces forêts. Ces chiffres nécessitent de revenir sur les méthodes qui permettent de calculer ces coûts.

\section{Les méthodes d'évaluation}

Les méthodes économiques engagées pour évaluer les coûts d'entretien et d'accroissement des services écologiques sont relativement simples sur le plan conceptuel, puisqu'elles visent, in fine, à calculer le coût des investissements qu'il est nécessaire de réaliser pour maintenir ou améliorer l'état de la biodiversité en vue de garantir la pérennité des flux de services écologiques. Ces investissements peuvent être de différentes natures : la restauration d'un écosystème qui a été dégradé ; la création d'un nouvel écosystème dans le cas de mesures compensatoires ; l'amélioration de la qualité d'un écosystème qui n'a pas été dégradé, mais dont les potentialités écologiques peuvent être accrues par un aménagement particulier, voire la préservation totale d'un écosystème ; mais aussi tous les coûts associés aux changements d'activités, de techniques, de pratiques qui ont pour objectif d'atténuer les impacts de ces dernières sur la biodiversité.

La principale particularité de ces méthodes est que des indicateurs physiques, en habitats, en espèces ou en services écologiques, permettent de s'assurer du maintien des potentialités écologiques.

À titre d'illustration, on peut mentionner la procédure américaine "Natural Resource Damage Assessment », qui s'appuie sur le principe de «no net loss » et qui oblige le responsable d'une pollution à mettre en œuvre des actions de restauration de manière à ce que toute perte de biodiversité et de services écosystémiques associée à la pollution soit compensée par un gain équivalent (Dunford et al., 2004 ; Bas et Gaubert, 2010). Le respect de ce principe nécessite d'avoir recours à des critères d'équivalence, comme l'illustre la figure 3. Le premier diagramme offre une représentation de l'impact d'un dommage environnemental sur un écosystème. Cet impact réduit les capacités de l'écosystèmeà délivrer le flux de services écologiques qu'il produit lorsqu'il est en bon état. L'impact est symbolisé par la courbe noire continue, qui représente la totalité des pertes en services écologiques pendant une période donnée. Ce dommage, en accord avec la loi américaine, doit être réparé physiquement par le responsable de l'incident. C'est pourquoi il nécessite un investissement dans un travail de restauration - dite « primaire »sur le site où il a eu lieu. L'objectif est d'accélérer le renouvellement des capacités de production de services écologiques par la biodiversité. Les résultats de cet investissement sont représentés par la courbe en pointillés. La surface B symbolise l'ensemble des services écologiques qui auraient été perdus si la procédure de restauration primaire n'avait pas été enclenchée.

Il reste cependant une perte résiduelle de services écologiques liée au dommage : la quantité de services perdus pendant la phase où l'impact del'incident a perduré, symbolisée par la surface A. Pour que la procédure de restauration soit complète, il faut qu'il y ait une compensation de ce dommage résiduel. Cela peut être réalisé dans un autre site où il est possible d'augmenter le niveau de production de services écologiques à partir d'un travail de restauration dite "compensatoire». L'effet de cet investissement est symbolisé, dans le deuxième diagramme, par la courbe en pointillés, qui permet de souligner un gain dans le niveau de production de services écologiques. L'objectif final est, schématiquement, que les surfaces A et $C$ s'équivalent. L'investissement correspondant au coût de maintien du flux de services écologiques est donc la somme des coûts de restauration, primaire et compensatoire, ainsi que de ceux associés au suivi des résultats de la procédure. Ces coûts ont pour origine la mobilisation de capital humain, naturel et physique dans le processus de restauration et de compensation, et non pas la valeur reconnue aux services écologiques perdus en raison de l'érosion, comme présenté dans la partie précédente.

Ces évaluations monétaires du coût de l'érosion de la biodiversité et des services écologiques s'inscrivent donc conceptuellement dans un cadre d'analyse coûtefficacité. L'efficacité pouvant être mesurée à l'aune de critères écologiques, mais aussi économiques, sociaux ou juridiques.

Ainsi, la figure 3 peut être utilisée dès que des normes de référence concernant un niveau de biodiversité ou de 


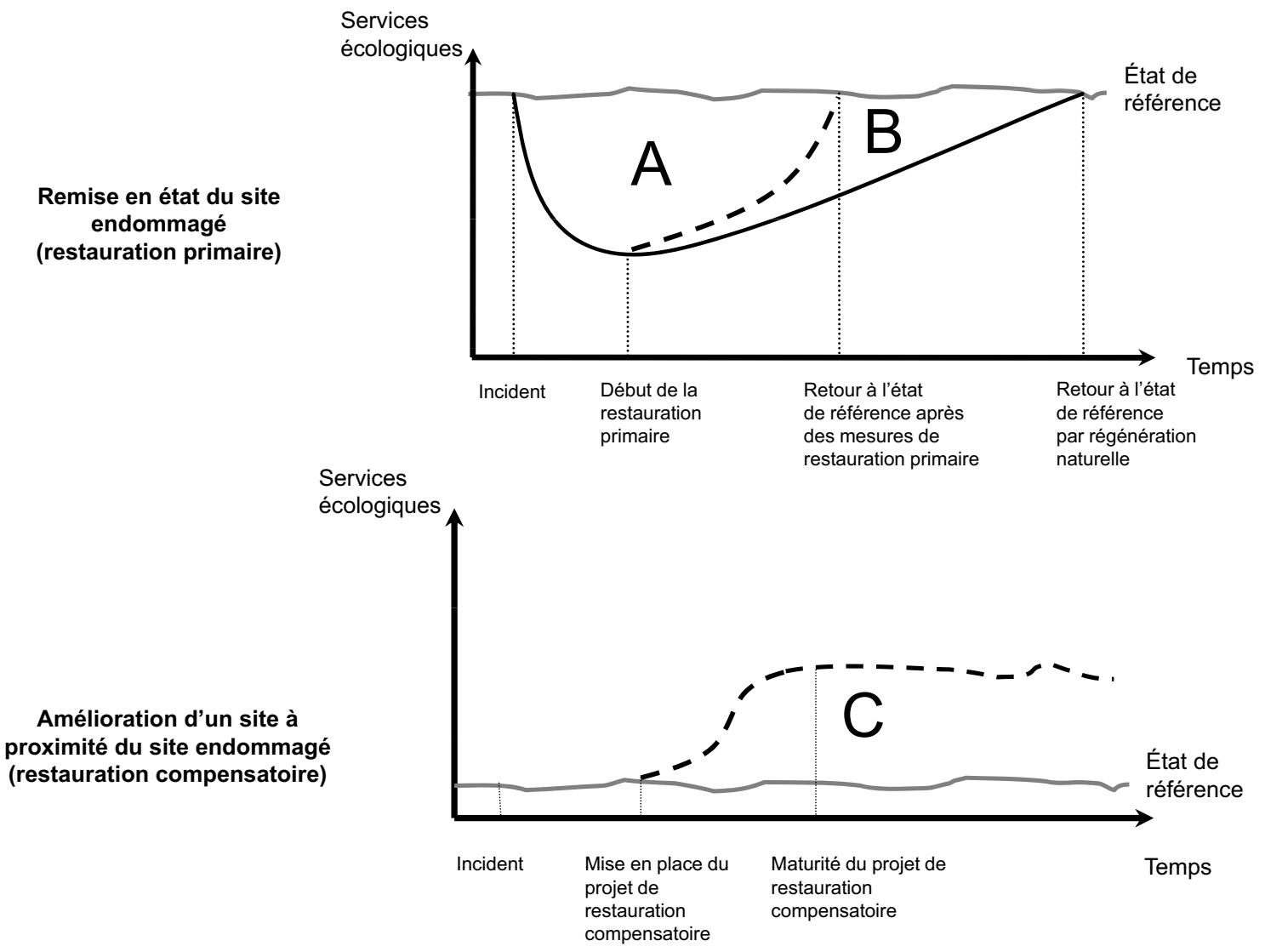

Fig. 3. Illustration des équivalents de services écologiques à restaurer et compenser dans le cadre des mesures «Natural Resource Damage Assessment » (source : Dunford et al., 2004).

services écologiques à maintenir ou à atteindre sont identifiées. Ces normes peuvent être, par exemple, celles de la directive-cadre sur l'eau et de la directive-cadre stratégie pour le milieu marin, qui fixent pour objectif aux États membres de l'Union européenne d'atteindre le bon état écologique de leurs eaux terrestres et de leurs milieux marins. Il s'agit dans ces cas de partir d'un état initial considéré comme désirable (au regard de la norme) et d'identifier les efforts et les moyens à mobiliser pour parvenir au respect de cette norme écologique, que cela se traduise par la réparation d'un dommage environnemental, la renaturalisation d'un écosystème, une évolution des usages ou encore la mise en place de mesures de prévention ou de techniques en vue de réduire les pressions anthropiques.

\section{Implication opérationnelle de l'usage des coûts de maintien des services écologiques pour l'évaluation des coûts de l'érosion de la biodiversité}

Aussi simple puisse-t-elle paraître, l'approche des coûts de maintien des services écologiques pour l'évaluation des coûts de l'érosion de la biodiversité se heurte cependant à plusieurs difficultés empiriques, essentielle- ment liées aux critères retenus pour qualifier l'efficacité des mesures permettant le maintien des potentialités.

Une première limite concerne la représentation graphique proposée dans la figure 3 et la référence à une forme d'équilibre symbolisé par la ligne grise. En effet, les écosystèmes ne connaissent pas d'équilibre à proprement parler. Par ailleurs, tout travail de restauration ou de compensation fera prendre à l'écosystème concerné une trajectoire différente de celle d'un écosystème naturel (Thanner et al., 2006). Il est donc impossible de considérer que les mesures de restauration ou de compensation permettront de faire aussi bien que la nature elle-même, ni que l'état écologique « désirable » recouvré sera comparable à l'état écologique initial. On peut néanmoins interpréter cette ligne grise comme le niveau de potentialités dont dispose l'écosystème pour délivrer des services écologiques.

Il est dès lors essentiel de savoir quelles sont les composantes de la biodiversité et des services écologiques qui ont été détruites, recréées et substituées, afin de pouvoir proposer un diagnostic précis des potentialités écologiques réellement maintenues. De ce point de vue, il apparaît important d'établir des critères d'efficacité 
écologique permettant de rendre plus objectifs les indicateurs qui guident les mesures de restauration et de compensation (Moberg et Rönnbäck, 2003 ; Palmer et Filoso, 2009).

Deux critiques principales sont formulées aujourd'hui à l'égard des indicateurs susceptibles d'évaluer l'efficacité des mesures de restauration et de compensation. La première est que ces indicateurs se focaliseraient sur un service écologique particulier sans tenir compte des interactions existant avec les autres services écologiques (Palmer et Filoso, 2009). Or, ces interactions peuvent être négatives ou positives et structurer fortement le succès des actions. La seconde critique est que les métriques retenues ne tiendraient pas compte des processus écologiques qui sont à l'origine du bon fonctionnement des écosystèmes, mais seulement de la production de services écologiques à court terme (Moberg et Rönnbäck, 2003). Cette production peut alors s'avérer non pérenne si les mécanismes qui en sous-tendent le maintien n'ont pas été réellement restaurés.

Plus généralement, les coûts associés à l'investissement dans la biodiversité varient fortement en fonction des niveaux de capital humain, physique et naturel à mobiliser, des contextes réglementaires et biophysiques dans lesquels ont lieu les investissements, mais surtout des objectifs écologiques préalablement fixés. Cela explique pourquoi la question des indicateurs d'efficacité destinés à évaluer les coûts doit être traitée de manière précise, dans un contexte de grande transparence. Le tableau 1 propose un gradient des équivalences permettant de qualifier l'efficacité des mesures compensatoires en précisant ce qui peut être entendu par « no net loss ». Le coût du maintien des potentialités écologiques dépendra ainsi du critère d'équivalence retenu (Mazzotta et al., 1994).

\section{Discussion : différences entre les coûts de l'érosion des services écologiques et de la biodiversité}

Il est possible de résumer les différences qui existent entre les deux méthodes d'évaluation des coûts de l'érosion des services écologiques à partir de quelques critères (cf. Tab. 2).

Comme nous l'avons vu, l'approche retenue par le TEEB se focalise sur la valeur de la biodiversité et, plus précisément, sur la perte de bénéfices potentiels générée par l'inaction politique dans le domaine de la conservation. Ainsi, le fait de ne pas avoir préservé la biodiversité depuis 2000 représente un coût estimé à 545 milliards de dollars à l'horizon 2010 et à près de 14000 milliards à l'horizon 2050 (Braat et ten Brink, 2008).

Une méthode d'évaluation alternative aurait pu être d'estimer les coûts de restauration, de compensation, d'innovation technique et de changements d'usages requis pour maintenir le flux des services écologiques à l'horizon 2010 ou 2050, sous l'hypothèse que l'objectif de ralentissement de l'érosion de la biodiversité fixé par la Convention sur la diversité biologique, voire l'objectif d'arrêt de cette érosion adopté par l'Union européenne, soit respecté à cet horizon. Dans ce cas, le coût de l'inaction est celui que les sociétés humaines auront à supporter après 50 ans d'inaction, si l'on considère qu'elles devront bien respecter, in fine, l'objectif d'arrêt de l'érosion de la biodiversité. Et il est possible de mettre en regard un tel coût avec ce qu'il en coûterait d'agir maintenant, en 2020 ou en 2030. L'idée est ici de souligner que plus on attend, plus la facture sera lourde, si l'on considère que la conservation de la biodiversité est bien un choix de société qui devra être assumé tôt ou tard. Cela apparaît assez évident lorsque l'on songe à l'exemple des espèces invasives (Pimmentel et al., 2005) et aux irréversibilités qui apparaissent, de manière générale, quand on laisse un écosystème se dégrader au point de le voir basculer dans un nouveau domaine de stabilité - moins productif et moins diversifié -, sans possibilité de retour sinon à un coût prohibitif (Gunderson et Holling, 2002).

Adopter une telle approche présente, de notre point de vue, deux avantages. Elle permet tout d'abord de se positionner dans un contexte de durabilité forte, car elle implique d'avoir des équivalences en unités physiques et consacre une logique d'action en faveur de la Nature afin d'enrayer sa dégradation (Roach et Wade, 2006). Ensuite, elle repose sur des méthodes d'évaluation permettant de mieux appréhender les fonctionnalités écologiques à l'origine des bénéfices non marchands et offre ainsi un outil de preuve plus convaincant pour les négociations autour des dommages causés à la biodiversité (Thompson, 2002).

Le passage à une échelle macroéconomique implique cependant de pouvoir mettre en place des méthodes d'évaluation des coûts de maintien des services écologiques à des échelles spatiales relativement larges (Weber, 2007). À ce titre, il pourrait être intéressant de savoir si une approche en termes de « transferts de coûts » intégrerait moins de variabilité dans les résultats obtenus que la méthode des «transferts de bénéfices ». En effet, dans le cas des transferts de coûts, la méthode de calcul est basée sur des processus de restauration associés à des connaissances en ingénierie écologique qui nécessitent des compétences humaines et techniques dont on peut objectivement mesurer les portées et les limites. Les coûts seront ainsi dépendants des moyens mobilisés pour produire les services écologiques, mais aussi de contraintes géophysiques et réglementaires précises dont on peut facilement connaître les caractéristiques. Ce n'est pas le cas des transferts de bénéfices, qui s'appuient sur des estimations très sensibles à un grand nombre de variables relevant du contexte socioéconomique, dont la 
Tableau 1. Les différents niveaux d'équivalence pour évaluer les compensations d'un point de vue économique*.

\begin{tabular}{|c|c|c|c|c|}
\hline & Niveau d'équivalence & Description & Exemple & Niveau de durabilité \\
\hline 1 & $\begin{array}{l}\text { Perte nette de biodiversité et de } \\
\text { bien-être }\end{array}$ & $\begin{array}{l}\text { Le capital naturel est détruit sans } \\
\text { compensation }\end{array}$ & $\begin{array}{l}\text { Prise accidentelle de } \\
\text { mammifères marins par les } \\
\text { pêcheurs }\end{array}$ & $\begin{array}{l}\text { Pas durable, car perte } \\
\text { nette }\end{array}$ \\
\hline 2 & $\begin{array}{l}\text { Pas de perte nette de bien-être } \\
\text { humain }\end{array}$ & $\begin{array}{l}\text { Le capital naturel détruit est } \\
\text { substitué par du capital physique } \\
\text { ou humain avec un niveau debien- } \\
\text { être humain équivalent }\end{array}$ & $\begin{array}{l}\text { Remplacement d'un herbier } \\
\text { marin (capital naturel) par } \\
\text { une infrastructure portuaire } \\
\text { (capital physique) }\end{array}$ & $\begin{array}{l}\text { Durabilité faible } \\
\text { (Pearce et Atkinson, } \\
1993 \text { ) }\end{array}$ \\
\hline 3 & $\begin{array}{l}\text { Pas de perte nette de capital } \\
\text { naturel, mais perte possible de } \\
\text { catégories de services } \\
\text { écosystémiques }\end{array}$ & $\begin{array}{l}\text { Le capital naturel détruit est } \\
\text { compensé ou remplacé par du } \\
\text { capital naturel produisant des } \\
\text { services écosystémiques différents }\end{array}$ & $\begin{array}{l}\text { Remplacement des services } \\
\text { de régulation produits par } \\
\text { l'herbier marin par des ser- } \\
\text { vices d'approvisionnement } \\
\text { liés à l'aquaculture }\end{array}$ & $\begin{array}{l}\text { Durabilité forte } \\
\text { (critère minimum) }\end{array}$ \\
\hline 4 & $\begin{array}{l}\text { Pas de perte nette de services de } \\
\text { régulation, deservices récréatifs, } \\
\text { de services de supports ni de } \\
\text { services d'approvisionnement, } \\
\text { mais substitution possible entre } \\
\text { services au sein de ces catégories }\end{array}$ & $\begin{array}{l}\text { Le capital naturel détruit est } \\
\text { compensé par du capital naturel } \\
\text { produisant les mêmes catégories } \\
\text { de services écosystémiques }\end{array}$ & $\begin{array}{l}\text { Les services de nourricerie } \\
\text { perdus du fait de la } \\
\text { destruction d'un herbier } \\
\text { marin peuvent être } \\
\text { compensés par la mise en } \\
\text { place de récifs d'huîtres } \\
\text { produisant un service de } \\
\text { filtration de l'eau (à niveau } \\
\text { constant de services de } \\
\text { régulation) }\end{array}$ & $\begin{array}{l}\text { Durabilité forte } \\
\text { (critère } \\
\text { intermédiaire) }\end{array}$ \\
\hline 5 & $\begin{array}{l}\text { Pas de perte nette de services } \\
\text { écosystémiques (sur la base des } \\
23 \text { services du MA, par exemple) }\end{array}$ & $\begin{array}{l}\text { Le capital naturel détruit est } \\
\text { compensé par du capital naturel } \\
\text { produisant les mêmes services } \\
\text { écosystémiques }\end{array}$ & $\begin{array}{l}\text { Lesservices denourricerie, de } \\
\text { filtration de l'eau, de } \\
\text { limitation de l'érosion, } \\
\text { associés aux herbiers marins } \\
\text { sont tous pris en compte et } \\
\text { sont individuellement } \\
\text { compensés }\end{array}$ & $\begin{array}{l}\text { Durabilité forte } \\
\text { (critère élevé) [Ekins, } \\
\text { 2003] }\end{array}$ \\
\hline 6 & $\begin{array}{l}\text { Le capital naturel est considéré } \\
\text { comme non substituable }\end{array}$ & $\begin{array}{l}\text { Le capital naturel ne peut être } \\
\text { détruit }\end{array}$ & & $\begin{array}{l}\text { Pas durable, car pas } \\
\text { de développement } \\
\text { possible }\end{array}$ \\
\hline
\end{tabular}

* Il s'agit en effet d'une description limitée des mesures d'équivalence, puisque la question de la biodiversité est envisagée ici uniquement sous un angle instrumental. Ainsi, l'échelle d'équivalence est beaucoup plus exigeante si l'on adopte une base taxonomique pour le calcul (ce qui est souvent le cas, puisqu'une part importante des mesures compensatoires vise des espèces protégées). Par ailleurs, les questions de la localisation géographique et de l'histoire des destructions et des compensations ne sont pas non plus prises en compte dans ce tableau, alors que ces éléments sont essentiels pour qualifier la pertinence des équivalences.

connaissance est complexe et nécessite la réalisation d'enquêtes relativement coûteuses. On notera toutefois que les coûts de maintien des services écologiques seront largement dépendants des indicateurs retenus pour qualifier les pertes et les gains ainsi que les niveaux d'équivalence permettant de considérer que le critère de «no net loss » est respecté ${ }^{6}$ (Tab. 1).

Si l'on retient comme indicateurs de pertes et de gains des espèces à court cycle de vie et des services de prélèvement, on pourra vraisemblablement restaurer et compenser les dommages à un plus faible coût que si l'on avait retenu des espèces à long cycle de vie ou des services de régulation, plus complexes et longs à obtenir. Par ailleurs, si le niveau d'équivalence exigé pour respecter le principe de «no net loss » est l'unité de capital naturel,

\footnotetext{
6 On le voit très bien aujourd'hui avec l'exemple des « mitigation banking » américaines concernant les zones humides, où les mesures compensatoires s'avèrent relativement inefficaces à faire respecter le principe du «no net loss » du fait de stratégies de compensation orientées par des critères plus économiques qu'écologiques (National Research Council, 2001).
} 
Tableau 2. Bénéfices potentiels versus coûts de maintenance : différence des approches.

\begin{tabular}{|c|c|c|}
\hline & Coût de maintenance des services écologiques & Valeur des services écologiques \\
\hline Principe général & $\begin{array}{l}\text { Quel est le coût des mesures positives pour } \\
\text { l'environnement que l'on doit consentir pour } \\
\text { restaurer et maintenir les services écologiques? }\end{array}$ & $\begin{array}{l}\text { Quelle est la valeur monétaire des pertes ou des } \\
\text { gains de bien-être associés aux évolutions des } \\
\text { services écologiques? }\end{array}$ \\
\hline $\begin{array}{l}\text { Champ d'application } \\
\text { principal }\end{array}$ & $\begin{array}{l}\text { Réglementation autour de la responsabilité } \\
\text { environnementale }\end{array}$ & Analyse financière pour la gestion de projet \\
\hline Évaluation des coûts & Coûts comptables & Coûts d'opportunité \\
\hline Cible & $\begin{array}{l}\text { L'environnement naturel en premier lieu et, } \\
\text { indirectement à travers lui, le bien-être des } \\
\text { acteurs bénéficiant de cet environnement }\end{array}$ & $\begin{array}{l}\text { Le bien-être des acteurs subissant des } \\
\text { externalités négatives }\end{array}$ \\
\hline Échelle économique & $\begin{array}{l}\text { Macroéconomique (valorisation à l'échelle du } \\
\text { socioécosystème) }\end{array}$ & $\begin{array}{l}\text { Microéconomique (valeurs individuelles } \\
\text { extrapolées au collectif) }\end{array}$ \\
\hline Unités d'équivalence & $\begin{array}{l}\text { Unités physiques (habitat, espèce, service } \\
\text { écologique) actualisées }\end{array}$ & $\begin{array}{l}\text { Unités de valeur (utilité, prix, bien-être) } \\
\text { actualisées }\end{array}$ \\
\hline $\begin{array}{l}\text { Théorie du capital } \\
\text { sous-jacente }\end{array}$ & Capital naturel critique & Épargne véritable \\
\hline $\begin{array}{l}\text { Méthode d'évaluation à de } \\
\text { larges échelles }\end{array}$ & Transfert de coûts & Transfert de bénéfices \\
\hline Principe de durabilité & $\begin{array}{l}\text { Forte à moyenne : la perte de capital naturel ne } \\
\text { peut être compensée que par du capital naturel } \\
\text { (faible substituabilité des différentes formes de } \\
\text { capital). Cependant, le niveau de compensation } \\
\text { peut fortement varier en fonction des indica- } \\
\text { teurs d'équivalence retenus }\end{array}$ & $\begin{array}{l}\text { Faible à moyenne : la perte de capital naturel } \\
\text { peut être compensée par du capital naturel, } \\
\text { humain ou physique (forte substituabilité des } \\
\text { différentes formes de capital). Cependant, la } \\
\text { prise en compte d'un niveau de capital naturel } \\
\text { critique peut permettre de nuancer cela }\end{array}$ \\
\hline
\end{tabular}

l'ensemble des mesures compensatoires possibles sera plus large que si l'on avait retenu l'unité de service écosystémique (Tab. 1). Il en résultera là encore un coût moindre des compensations.

Plus globalement, les choix arrêtés, relatifs aux indicateurs et aux niveaux d'équivalence, vont conduire à définir le domaine de durabilité dans lequel on envisage le maintien des potentialités écologiques, avec pour corollaire les coûts à supporter pour ce maintien.

Si l'analyse des avantages et des limites de ces deux approches complémentaires est nécessaire, elle ne doit pas faire oublier qu'en pratique, la pertinence d'une méthode d'évaluation des coûts de l'érosion de la biodiversité dépend également du contexte institutionnel dans lequel cette évaluation s'inscrit. À titre d'exemple, pour ce qui concerne les dommages environnementaux, l'approche par les coûts de maintien de la biodiversité semble trouver depuis quelques années, aux États-Unis, un écho et une adhésion plus larges que l'approche par les pertes de valeurs (Thompson, 2002).

On observe une tendance similaire en Europe, comme en témoigne la mention de cette méthode dans plusieurs textes réglementaires récents. Ainsi, la référence aux services écologiques et à leur coût de maintien a été intégrée dans le droit français (article L.161-1 du Code de l'environnement) avec la Directive européenne 2004/35/CE relative à la responsabilité environnementale et la loi de transposition $\mathrm{n}^{\circ} 2008-757 \mathrm{du} 1^{\mathrm{er}}$ août 2008, dont le décret $n^{\circ} 2009-468$ a été publié le 23 avril 2009 : «Constituent des dommages causés à l'environnement au sens du présent titre les détériorations directes ou indirectes mesurables de l'environnement qui : [...] Affectent les services écologiques, c'est-à-dire les fonctions assurées par les sols, les eaux et les espèces et habitats mentionnés au $3^{\circ}$ au bénéfice d'une de ces ressources naturelles ou au bénéfice du public [...]. » L'article L.162-11 précise par ailleurs : «Les mesures de réparation des dommages [...] visent à rétablir ces ressources naturelles et leurs services écologiques dans leur état initial [...]. L'état initial désigne l'état des ressources naturelles et des services au moment du dommage, qui aurait existé si celui-ci n'était pas survenu. »Enfin, 1'article L.162-20 souligne que le responsable des dommages devra supporter les frais liés « $1^{\circ}$ À l'évaluation 
des dommages ; $2^{\circ}$ À la détermination, la mise en œuvre et le suivi des mesures de prévention et de réparation $[\ldots] »$. Cette loi stipule aussi l'obligation de réparer les « dommages écologiques purs » et interdit leur réparation par équivalent monétaire, sauf cas exceptionnel.

La question de l'évaluation des coûts de maintien des services écologiques se pose aussi dans le cadre des évaluations d'impacts de projets d'aménagement, dont le Code de l'environnement précise le contenu à travers son article L.122-1 : lesétudes d'impacts doivent intégrer « les mesures envisagées pour supprimer, réduire et, si possible, compenser les conséquences dommageables pour l'environnement et la santé ».

\section{Conclusion}

L'objet de ce papier était de clarifier la notion de coût de l'érosion de la biodiversité. Un premier résultat mis en avant est qu'il y a une double acception de ce concept : d'un côté, le coût représente une perte de valeur associée à l'érosion de la biodiversité ; de l'autre, les investissements nécessaires au maintien des potentialités écologiques. Ces deux approches renvoient chacune à des enjeux théoriques et des méthodes d'évaluation à la fois différents et complémentaires.

Depuis plusieurs décennies, l'approche économique de la biodiversité a privilégié une analyse des coûts en termes de valeur. Malgré la richesse des débats académiques autour de ce thème, on constate une certaine frustration du point de vue de la portée opérationnelle de ces travaux, du fait d'un manque de données, mais aussi de méthodes d'évaluation indirectes, relativement coûteuses à mettre en œuvre.

Pour résumer, les travaux sur cette question peuvent être séparés selon deux options empiriques. La première option est de limiter l'évaluation à une partie des services écologiques fournis par la biodiversité dans un contexte socioéconomique précis. Cette attitude prudente est celle reprise, par exemple, dans le rapport du CAS (Chevassusau-Louis et al., 2009). Ces résultats sont robustes, mais ils ne représentent évidemment qu'une faible partie de la valeur des services écologiques et de la biodiversité. La seconde option consiste à évaluer la valeur économique totale des services écologiques délivrés par la biodiversité, au moyen de méthodes simplistes et souvent grossières - c'est le cas du rapport intermédiaire du TEEB sur les coûts de l'inaction politique (Braat et ten Brink, 2008) ou des travaux de Costanza et al. (1997), qui ont largement recours aux méthodes de transferts de bénéfices. Dans chacune des deux démarches, le public ou le décideur peut ressentir une certaine frustration: soit par le caractère partiel du résultat obtenu, soit par le manque de robustesse de la méthode adoptée.

L'approche par les coûts de maintien de la biodiversité bénéficie d'un intérêt plus récent de la part des économistes, en partie du fait de la frustration mentionnée cidessus et de la montée en puissance du principe de durabilité forte, notamment dans les textes réglementaires. Elle a pour avantage de dépasser les problèmes rencontrés dans l'évaluation monétaire non marchande, en s'appuyant sur des critères d'équivalence physique offrant de meilleures garanties de respecter ce critère de durabilité forte. Pour autant, cette approche nécessite d'être questionnée dans les années à venir, notamment du fait des critères d'efficacité écologique, qui conditionnent la pertinence de sa mise en œuvre et la portée de l'évaluation obtenue.

\section{Remerciements}

Nous tenons à remercier Denis Bailly, Rémi Mongruel, José Pérez et Anne-Charlotte Vaissière pour les discussions que nous avons pu avoir avec eux sur le sujet des coûts de l'érosion de la biodiversité et des services écologiques.

\section{Références}

Banque mondiale, 1997. Expanding the Measure of Wealth: Indicators of Environmentally Sustainable Development, Washington (DC), Banque mondiale.

Barbier, E.B., 1994. Valuing environmental functions, tropical wetlands, Land Economics, 70, 155-173.

Barbier, E.B., Baumgärtner, S., Chopra, K., Costello, C., Duraiappah, A., Hassan, R., Kinzig, A., Lehmann, M., Pascual, U., Polasky, S., Perrings, C., 2009. The Valuation of ecosystem services, in Naeem, S., Bunker, D.E., Hector, A., Loreau, M., Perrings, C. (Eds), Biodiversity, Ecosystem Functioning, and Human Well Being, Oxford and New York, Oxford University Press, 248-262.

Bartelmus, P., 2009. The cost of natural capital consumption: Accounting for a sustainable world economy, Ecological Economics, 68, 6, 1850-1857.

Bas, A., Gaubert, H., 2010. La Directive « Responsabilité environnementale» et ses méthodes d'équivalence, La Défense, Commissariat général du développement durable, Service de l'économie, de l'évaluation et de l'intégration du développement durable (http:/ / www.developpement-durable. gouv.fr/IMG/pdf/ED19b.pdf).

Boltanski, L., Thévenot, L., 1991. De la justification : les économies de la grandeur, Paris, Gallimard. 
Borde, A.B., O'Rourke, L.K., Thom, R.M., Williams, G.W., Diefenderfer, H.L., 2004. National Review of Innovative and Successful Coastal Habitat Restoration. Report prepared for National Oceanic and Atmospheric Administration, Coastal Services Center, Battelle Memorial Institute, Duxbury (MAS).

Braat, L., ten Brink, P., 2008. The Cost of Policy Inaction (COPI): The Case of not Meeting the 2010 Biodiversity Target. Report to the European Commission, Wageningen/Brussels (http:/ / ec.europa.eu/environment/nature/biodiversity/ economics/teeb_en.htm).

Chevassus-au-Louis, B., Salles, J.-M., Pujol, J.-L. (Eds), Conseil d'analyse stratégique, 2009. Approche économique de la biodiversité et des services liés aux écosystèmes, Paris, La Documentation française.

Commission des comptes et de l'économie de l'environnement, 2009. L'Économie de l'environnement en 2007 : rapport de la Commission des comptes et de l'économie de l'environnement. Édition 2009. Commissariat général au développement durable, Service de l'observation des statistiques, La Défense.

Committee on Assessing and Valuing the Services of Aquatic and Related Terrestrial Ecosystems, National Research Council, 2004. Valuing Ecosystem Services: Toward Better Environmental Decision-Making, Washington (DC), The National Academies Press.

Costanza, R., d'Arge, R., de Groot, R., Farber, S., Grasso, M., Hannon, B., Limburg, K., Naeem, S., O'Neill, R.V., Paruelo, J., Raskin, R.G., Sutton, P., van den Belt, M., 1997. The value of the world's ecosystem services and natural capital, Nature, 387, 253-260.

Costanza, R., Fisher, B., Mulder, K., Liu, S., Christopher, T., 2007. Biodiversity and ecosystem services: A multi-scale empirical study of the relationship between species richness and net primary production, Ecological Economics, 61, 2-3, 478-491.

Daily, G.C., Polasky, S., Goldstein, J., Kareiva, P.M., Mooney, H.A., Pejchar, L., Ricketts, T.H., Salzman, J., Shallenberger, R., 2009. Ecosystem services in decision making: Time to deliver, Frontiers in Ecology and the Environment, 7, 1, 21-28.

Desrosières, A., 2003. Les qualités des quantités, Courrier des statistiques, 105-106, 51-63.

Dunford, R.W., Ginn, T.C., Desvousges, W.H., 2004. The use of habitat equivalency analysis in natural resource damage assessments, Ecological Economics, 48, 1, 49-70.

Ekins, P., 2003. Identifying critical natural capital: Conclusions about critical natural capital, Ecological Economics, 44, 2-3, 277-292.

Environmental Protection Agency, 2009. Valuing the Protection of Ecological Systems and Services. Report of the EPA Science Advisory Board, Washington (DC).

Espeland, W.N., Stevens, M.L., 1998. Commensuration as a social process, Annual Review of Sociology, 24, 313-343.

Fonseca, M.S., Kenworthy, W.J., Julius, B.E., Shutler, S., Fluke, S., 2002. Seagrasses, in Perrow, M.R., Davy, A.J., Handbook of Ecological Restoration, vol. 2, Cambridge (UK) and New York, Cambridge University Press, 149-170.
Gunderson, L.H., Holling, C.S. (Eds), 2002. Panarchy: Understanding Transformations in Human and Natural Systems, Washington (DC), Island Press.

Heal, G., 2000. Nature and the Marketplace: Capturing the Value of Ecosystem Services, Washington (DC), Island Press.

Jones, C.-A., Pease, K.-A., 1997. Restoration-based compensation measure in natural resource liability statutes, Contemporary Economic Policy, 15, 110-122.

Kalaydjian, R. (Ed.), 2007. La Mer a de l'avenir: données économiques maritimes françaises 2007. Synthèse Ifremer, Issy-les-Moulineaux.

King, D.M., Bohlen, C.C., 1995. The Cost of Wetland Creation and Restoration. Technical Report DOE/MT/92006-9 (DE95000174), US Department of Energy, Washington(DC).

Krutilla, J.V., 1967. Conservation reconsidered, The American Economic Review, 57, 4, 83-91.

Levrel, H., Fontaine, B., Henry, P-Y, Jiguet, F., Julliard, R., Kerbiriou, C., Couvet, D., 2010. Balancing state and volunteer investment in biodiversity monitoring for the implementation of CBD indicators: A French example, Ecological Economics, 69, 7, 1580-1586.

Lewis, R.R., 2001. Mangrove restoration: Costs and benefits of successful ecological restoration, Mangrove Valuation Workshop, Universiti Sains Malaysia, Penang, 4-8 April, Beijer International Institute of Ecological Economics, Stockholm.

Martinez, M.L., Intralawan, A., Vázquez, G., Pérez-Maqueo, O., Sutton, P., Landgrave, R., 2007. The coasts of our world: Ecological, economic and social importance, Ecological Economics, 63, 2-3, 254-272.

Mazzotta, M., Opaluch, J.J., Grigalunas, T.A., 1994. Natural resource damage assessment: The role of resource restoration, Natural Resource Journal, 34, 1, 153-178.

Micklin, P., 2007. The Aral Sea disaster, Annual Review of Earth and Planetary Sciences, 35, 47-72.

Millennium Ecosystem Assessment, 2005. Ecosystems and Human Well-being: Synthesis, Washington (DC), Island Press.

Moberg, F., Rönnbäck P., 2003. Ecosystem services of the tropical seascape: Interactions, substitutions and restoration, Ocean Coastal Management, 46, 27-46.

National Research Council, 2000. Watershed Management for Potable Water Supply: Assessing the New York City Strategy, Washington (DC), The National Academy Press.

National Research Council, 2001. Compensating for Wetlands Losses under the Clean Water Act: Committee on Mitigating Wetland Losses, Washington (DC), The National Academy Press.

Nelson, E., Mendoza, G., Regetz, J., Polasky, S., Tallis, H., Cameron, D.R., Chan, K.M., Daily, G.C., Goldstein, J., Kareiva, P.M., Lonsdorf, E., Naidoo, R., Ricketts T., Shaw, M.R., 2009. Modeling multiple ecosystem services, biodiversity conservation, commodity production, and tradeoffs at landscape scales, Frontiers in Ecology and the Environment, 7, 1, 4-11.

Norgaard, R.B., Bode, C., 1998. Next, the value of God, and other reactions, Ecological Economics, 25, 1, 37-39. 
OCDE, 2004. Handbook of Market Creation for Biodiversity: Issues in Implementation, Paris, OECD Publications.

Palmer, M.A., Filoso, S., 2009. Restoration of ecosystem services for environmental markets, Science, 325, 575-576.

Pearce, D.W., Atkinson, G.D., 1993. Capital theory and the measurement of sustainable development: An indicator of "weak" sustainability, Ecological Economics, 8, 2, 103-108.

Pimentel, D., Zuniga, R., Morrison, D., 2005. Update on the environmental and economic costs associated with alieninvasive species in the United States, Ecological Economics, 52, 3, 273-288.

Roach, B., Wade, W.W., 2006. Policy evaluation of natural resource injuries using habitat equivalency analysis, Ecological Economics, 58, 2, 421-433.

Thanner, S.E., McIntosh, T.L., Blair, S.M., 2006. Development of benthic and fish assemblages on artificial reef materials compared to adjacent natural reef assemblages in MiamiDade county, Florida, Bulletin of Marine Science, 78, 1, 57-70.

The Economics of Ecosystem and Biodiversity, 2010. Mainstreaming the Economics of Nature: A synthesis of the Approach, Conclusions and Recommendations of TEEB (http:// www.teebweb.org/Portals/25/TEEB\%20Synthesis/ TEEB_Synth Report_09_2010_online.pdf).

Thompson, D.B., 2002. Valuing the environment: Courts' struggles with natural resource damages, Environmental Law, 32, 57-89.

Weber, J.-L., 2007. Implementation of land and ecosystem accounts at the European Environment Agency, Ecological Economics, 61, 4, 695-707.

Zafonte, M., Hampton, S., 2007. Exploring welfare implications of resource equivalency analysis in natural resource damage assessments, Ecological Economics, 61, 1, 134-145.

Reçu le 17 juin 2010. Accepté le 7 avril 2011. 